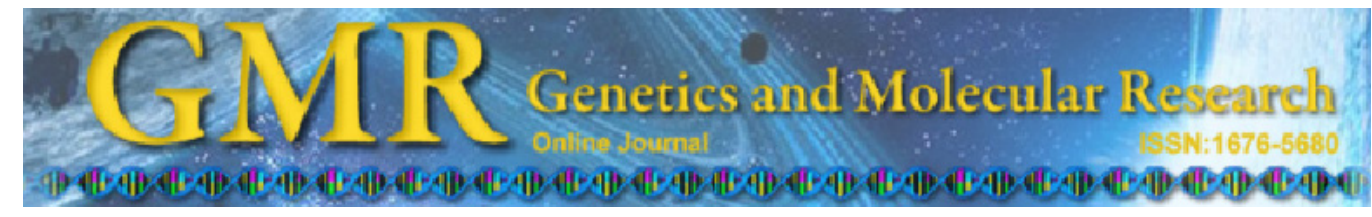

\title{
Correlation of Y-chromosome multiple segmental deletions and chromosomal anomalies in non-obstructive azoospermic males from northeastern China
}

\author{
R.-L. Dai ${ }^{1,2}$, R.-X. Wang ${ }^{1}$, J.-L. Jin ${ }^{3}$, G.-N. Niu ${ }^{3}$, J.-Y. Lee ${ }^{3}$, S.-B. Li ${ }^{3}$ and \\ R.-Z. Liu ${ }^{1}$ \\ ${ }^{1}$ Center for Reproductive Medicine, First Bethune Hospital, Jilin University, \\ Changchun, China \\ ${ }^{2}$ Department of Pathophysiology, Norman Bethune College of Medicine, \\ Jilin University, Changchun, China \\ ${ }^{3}$ Genetic Section, Department of Pediatrics, University of Oklahoma, \\ Health Sciences Center, Oklahoma, City, OK, USA \\ Corresponding author: R.-Z. Liu \\ E-mail: lanxin910@163.com
}

Genet. Mol. Res. 11 (3): 2422-2431 (2012)

Received September 16, 2011

Accepted January 24, 2012

Published May 10, 2012

DOI http://dx.doi.org/10.4238/2012.May.10.3

\begin{abstract}
We investigated the frequency and types of Y-chromosome microdeletions and chromosomal anomalies in non-obstructive azoospermic and severely oligozoospermic infertile males in northeastern China. The sample consisted of 519 infertile males (456 azoospermic, 63 severely oligozoospermic). PCR assays for Y-chromosome microdeletions and chromosome analysis were performed on all patients and controls. Array-comparative genomic hybridization was performed for three patients with chromosomal anomalies. Fifty-nine of 519 patients $(11.37 \%)$ had Y-chromosome microdeletions. Microdeletions were found in $11.18 \%(51 / 456)$ of the non-obstructive azoospermic patients and in $12.7 \%(8 / 63)$ of the severely oligozoospermic patients. Eleven of 51 non-obstructive azoospermic patients with Y-chromosome microdeletions had multiple segmental deletions in the $\mathrm{AZFb}+\mathrm{c}$ regions; four of these patients had chromosomal anomalies.
\end{abstract}


Our sample from northeastern China had a higher frequency of microdeletions among severely oligozoospermic than among non-obstructive azoospermic males.

Key words: Azoospermia; Array-comparative genomic hybridization; Severe oligozoospermia; Azoospermia factor; Chromosomal anomaly

\section{INTRODUCTION}

Infertility is a major health problem that affects approximately 10 to $15 \%$ of the reproductive-aged population. Male factors have been identified in approximately half of these cases (Balkan et al., 2008). The investigation of male infertility should include the identification and analysis of Y-chromosome microdeletions and chromosomal anomalies, the most frequent genetic causes of this condition (Tiepolo and Zuffardi, 1976).

Most microdeletions that cause azoospermia or oligozoospermia occur in the long (q) arm of the Y-chromosome. Within the Yq region, the azoospermia factor (AZF) region is divided into 3 subregions (AZFa-c) that are approximately 1.1, 3.2, and $3.5 \mathrm{Mb}$ in size, respectively (Briton-Jones and Haines, 2000; Gatta et al., 2002). A fourth subdivision (AZFd) located between AZFb and AZFc has been proposed (Kent-First et al., 1999) even though AZFd is a well-established part of the AZFc region. The genes in the AZF region are considered critical for spermatogenesis, and the frequency of microdeletions in the Yq region has been associated with the severity of spermatogenic defects (Krausz et al., 2001). This predisposition for infertility can also include gradual alterations in spermatozoa production such that men with oligozoospermia may later develop azoospermia (Kihaile et al., 2005).

Somatic chromosomal anomalies occur in approximately $10 \%$ of the infertile male population. These anomalies may be numeric or structural and may involve sex chromosomes or autosomes. The Y-chromosome is characterized by a high level of structural variability, including deletions, duplications, and inversions (Balaresque et al., 2008).

During the last decade, the ability to address male infertility has improved enormously owing to the development of assisted fertilization techniques, such as intracytoplasmic sperm injection. However, the underlying reasons for male infertility require further investigation. Because Y-chromosome microdeletions and chromosomal anomalies may be transmitted readily from infertile fathers to their offspring through intracytoplasmic sperm injection, the clinical diagnosis of the cause of infertility before the use of such techniques in important (Devroey et al., 2009). In the present study, we aimed to determine the frequency and types of Y-chromosome microdeletions in non-obstructive azoospermic and severely oligozoospermic infertile men in northeastern China and to investigate the relationship between these microdeletions and chromosomal karyotypes.

\section{MATERIAL AND METHODS}

\section{Patients}

We evaluated 540 infertile male patients aged 20-47 years who attended the outpatient infertility clinic of the andrology, urology, and gynecology departments of the First Bethune 
Hospital of Changchun, Jilin Province, northeastern China, between November 6, 2006 and August 31, 2010. This study was approved by the ethics committee of First Bethune Hospital, and all patients provided informed consent to participate in the study.

Cytogenetic analysis was performed for patients with azoospermia or severe oligozoospermia ( 1 year of infertility, at least three semen samples with sperm counts of $<5$ million $/ \mathrm{mL}$ ). Twenty-one of the 540 patients were diagnosed with obstructive azoospermia and excluded from the study. Thus, the final study sample consisted of 519 patients. Semen analysis was performed for all study participants according to the recommendations of the World Health Organization (Organization, 1999). The results showed that 456 patients had non-obstructive azoospermia (after centrifugation) and 63 had severe oligozoospermia.

\section{DNA and polymerase chain reaction (PCR) analyses}

Genomic DNA was isolated from peripheral blood lymphocytes using the Tiangen Blood DNA Extraction Mini Kit (Beijing Tiangen Biotech Co. Ltd., China). DNA samples obtained from normal men with proven fertility and normal women were used as positive and negative controls. A sample containing all reaction components and water was used as a blank control.

Based on the recommendations of the European Academy of Andrology and the European Molecular Genetics Quality Network, the patients were tested for the markers proposed by Simoni et al. (2004): sY84, sY86, sY127, sY134, sY143, sY152, sY254, and sY255, with sY14 (sex-determining region of the Y-chromosome) and sex-chromosomal zinc-finger genes $(\mathrm{ZFX} / \mathrm{ZFY})$ as internal controls. PCR was carried out in a total volume of $20 \mu \mathrm{L}$. A PCR thermal cycler (Veriti 96-well PCR; Applied Biosystems, Carlsbad, CA, USA) was used to complete the PCR amplification reaction. Each experiment was performed at least twice. Results were considered positive when a clear amplification product of the expected size was obtained. In cases of negative results, PCR was repeated two more times or until acceptable results were achieved.

\section{Karyotype analysis}

After culturing the peripheral blood samples for $72 \mathrm{~h}$, we obtained lymphocyte chromosome spreads using routine preparation methods (Shaffer et al., 2009). Karyotypes were described according to the International System for Chromosome Nomenclature (Shaffer et al., 2009) and analyzed after applying G-banding. For each individual, a minimum of 30 metaphase cells were counted and at least five cells were analyzed.

\section{Array-comparative genomic hybridization}

For oligonucleotide array comparative genomic hybridization (CGH), DNA was isolated from peripheral blood samples using a commercially available extraction kit (Puregene blood kit; QIAGEN Inc., Valencia, CA, USA) following the manufacturer recommended protocol. Oligonucleotide array-CGH was performed using a 385-k oligonucleotide chip (Roche NimbleGen System Inc., Madison, WI, USA), according to the manufacturer protocol with minor modifications. A commercially available, pooled, normal control DNA (Promega Cor- 
poration, Madison, WI, USA) was used for reference. Patient and reference DNA were labeled with cyanine 3 or cyanine 5 via random priming (TriLink Biotechnologies, San Diego, CA, USA) and then hybridized to the chip via incubation in a Micro Array User Interface hybridization system (BioMicro Systems, Salt Lake City, UT, USA). After 18 h of hybridization at $42^{\circ} \mathrm{C}$, the slides were washed and scanned using a GenePix 4000B (Molecular Devices, Sunnyvale, CA, USA). Data were analyzed using NimbleScan 2.4 and SignalMap 1.9 (NimbleGen Systems Inc., Madison, WI, USA).

\section{RESULTS}

\section{Clinical analysis}

Of the 519 male patients from northeastern China with non-obstructive infertility, 456 were azoospermic (after centrifugation) and 63 were severely oligozoospermic. Fiftynine patients $(11.37 \%, 59 / 519)$ had Y-chromosome microdeletions, of which $51(11.18 \%$, $51 / 456)$ were non-obstructive azoospermic and eight $(12.70 \%, 8 / 63)$ were severely oligozoospermic. The mean age of azoospermic patients was 32 years (range: $20-45$ years) and that of severely oligozoospermic patients was 31 years (range: $22-40$ years; Table 1). Amplification of the sex-determining region of the Y-chromosome and ZFX/ZFY genes were achieved in all patients and positive controls, whereas only the ZFX/ZFY gene was amplified in the negative control.

\section{Karyotype analysis and array-CGH}

Chromosome analyses were performed for 519 patients. Fifty-three patients $(10.21 \%$, 53/519) had chromosomal anomalies and 41 patients $(77.36 \%$, 41/53) had Klinefelter's syndrome $(47, \mathrm{XXY})$. Forty of the 456 non-obstructive azoospermic patients had Klinefelter's syndrome $(47, \mathrm{XXY})$; one of these patients exhibited the chromosomal mosaic Klinefelter's syndrome (46,XX[19]/47,XXdel[Y][q11][41]), and 9 had special chromosomal anomalies (chromosomal mosaicism, $\mathrm{N}=3$; structural aberrations, $\mathrm{N}=6$ ). Among the 63 severely oligozoospermic patients, one had a 47,XXY karyotype (Klinefelter's syndrome) and two had translocations (Table 2).

Array-CGH studies were performed on 2 non-obstructive azoospermic patients and 1 severely oligozoospermic patient with special chromosomal anomalies. The results of these analyses corresponded with those of the karyotype analyses (Table 3).

\section{Y-chromosome microdeletion analysis}

Among the 51 azoospermic patients with microdeletions, 1 patient had microdeletions in the AZFa region, 2 had microdeletions in the AZFb region, 34 had microdeletions in the $\mathrm{AZFc}$ region, and 14 had microdeletions in the $\mathrm{AZFb}+\mathrm{c}$ regions (Table 4). Eleven patients with microdeletions $(21.57 \%, 11 / 51)$ in the $\mathrm{AZFb}+\mathrm{c}$ regions showed multiple segmental deletions, and 4 of these patients $(7.84 \%, 4 / 51)$ had special chromosomal anomalies (Table 5). All of the 8 severely oligozoospermic patients had microdeletions in the AZFc regions (see Table 4). 


\begin{tabular}{|c|c|c|c|c|c|c|c|c|c|c|}
\hline \multirow[t]{2}{*}{ Patient deletion No. } & \multirow[t]{2}{*}{ Age (years) } & \multicolumn{2}{|c|}{ AZFa } & \multicolumn{3}{|c|}{$\mathrm{AZFb}$} & \multicolumn{4}{|c|}{$\mathrm{AZFc}$} \\
\hline & & sY86 & sY84 & sY127 & sY134 & sY143 & sY152 & sY254 & sY255 & sY157 \\
\hline \multicolumn{11}{|l|}{ Azoospermic } \\
\hline 1 & 30 & + & + & + & + & + & - & - & - & - \\
\hline 2 & 44 & + & + & - & - & + & - & - & - & + \\
\hline 3 & 26 & + & + & - & - & + & - & - & - & - \\
\hline 4 & 24 & + & + & + & + & + & - & - & - & - \\
\hline 5 & 33 & + & + & + & - & - & - & - & - & - \\
\hline 6 & 35 & + & + & - & - & + & - & - & - & - \\
\hline 7 & 30 & + & + & + & + & + & - & - & - & - \\
\hline 8 & 28 & + & + & + & + & + & - & - & - & - \\
\hline 9 & 24 & + & + & - & - & + & - & - & - & - \\
\hline 10 & 35 & + & + & + & + & + & - & - & - & + \\
\hline 11 & 37 & + & + & + & + & + & - & - & - & - \\
\hline 12 & 29 & + & + & + & + & + & - & - & - & - \\
\hline 13 & 28 & + & + & + & + & + & - & - & - & + \\
\hline 14 & 31 & + & + & + & + & + & - & - & - & - \\
\hline 15 & 35 & + & + & + & + & + & - & - & - & - \\
\hline 16 & 38 & + & + & + & + & + & + & - & - & - \\
\hline 17 & 30 & + & + & - & + & + & - & - & - & - \\
\hline 18 & 33 & - & - & + & + & + & + & + & + & + \\
\hline 19 & 37 & + & + & + & + & + & - & - & - & - \\
\hline 20 & 40 & + & + & + & + & + & - & - & - & - \\
\hline 21 & 40 & + & + & - & - & - & + & + & + & + \\
\hline 22 & 42 & + & + & + & - & + & - & - & - & - \\
\hline 23 & 45 & + & + & + & + & + & - & - & - & - \\
\hline 24 & 33 & + & + & + & + & + & - & - & - & - \\
\hline 25 & 30 & + & + & - & - & + & - & - & - & - \\
\hline 26 & 29 & + & + & - & + & + & - & - & - & - \\
\hline 27 & 25 & + & + & + & + & + & - & - & - & - \\
\hline 28 & 36 & + & + & + & + & + & - & - & - & - \\
\hline 29 & 38 & + & + & + & + & + & - & - & - & - \\
\hline 30 & 34 & + & + & - & + & + & - & - & - & - \\
\hline 31 & 33 & + & + & + & + & + & - & - & - & + \\
\hline 32 & 29 & + & + & - & - & + & - & - & - & + \\
\hline 33 & 44 & + & + & + & + & + & - & - & - & - \\
\hline 34 & 26 & + & + & + & + & + & - & - & - & - \\
\hline 35 & 28 & + & + & - & - & + & - & - & - & + \\
\hline 36 & 39 & + & + & + & - & - & - & - & - & - \\
\hline 37 & 26 & + & + & + & - & - & - & - & - & - \\
\hline 38 & 30 & + & + & + & + & + & - & - & - & - \\
\hline 39 & 32 & + & + & + & + & + & - & - & - & - \\
\hline 40 & 31 & + & + & + & + & + & - & - & - & - \\
\hline 41 & 40 & + & + & + & + & + & - & - & - & - \\
\hline 42 & 37 & + & + & + & + & + & - & - & - & - \\
\hline 43 & 35 & + & + & + & + & + & - & - & - & - \\
\hline 44 & 41 & + & + & + & + & + & - & - & - & - \\
\hline 45 & 26 & + & + & + & + & + & - & - & - & - \\
\hline 46 & 31 & + & + & + & + & + & + & - & - & - \\
\hline 47 & 30 & + & + & + & + & + & - & - & - & - \\
\hline 48 & 20 & + & + & - & - & - & + & + & + & + \\
\hline 49 & 27 & + & + & + & + & + & - & - & - & - \\
\hline 50 & 29 & + & + & + & + & + & - & - & - & - \\
\hline 51 & 21 & + & + & + & + & + & - & - & - & - \\
\hline \multicolumn{11}{|c|}{ Severely oligozoospermic } \\
\hline 52 & 22 & + & + & + & + & + & - & - & - & - \\
\hline 53 & 30 & + & + & + & + & + & - & - & - & + \\
\hline 54 & 28 & + & + & + & + & + & - & - & - & - \\
\hline 55 & 35 & + & + & + & + & + & - & - & - & - \\
\hline 56 & 33 & + & + & + & + & + & - & - & - & - \\
\hline 57 & 29 & + & + & + & + & + & - & - & - & - \\
\hline 58 & 31 & + & + & + & + & + & - & - & - & - \\
\hline 59 & 40 & + & + & + & + & + & - & - & - & - \\
\hline
\end{tabular}

$(-)=$ Sequence-tagged site marker deleted; $(+)=$ sequence-tagged site marker present. 
Table 2. Summary of abnormal karyotypes other than non-mosaic Klinefelter's syndrome.

\begin{tabular}{ll}
\hline No. & Abnormal karyotype \\
\hline Azoospermic & \\
12 & $45, \mathrm{X},-\mathrm{Y},-13,+\mathrm{t}(\mathrm{Y} ; 13)(\mathrm{p} 1 ; \mathrm{q} 10)$ \\
72 & $46, \mathrm{XX}[19] / 47, \mathrm{XXdel}(\mathrm{Y})(\mathrm{q} 11)[41]$ \\
98 & $45, \mathrm{X},-\mathrm{Y},-15,+\mathrm{t}(\mathrm{Y} ; 15)(\mathrm{p} ? ; \mathrm{q} 11)$ \\
167 & $46, \mathrm{XY}, \mathrm{del}(\mathrm{Yq})$ \\
241 & $46, \mathrm{XY}[51] / 45, \mathrm{X}[9](\mathrm{Y}<\mathrm{G})$ \\
267 & $46, \mathrm{XY}, \mathrm{t}(1 ; 2)(\mathrm{q} 21 ; \mathrm{p} 23)$ \\
274 & $46, \mathrm{XY}[34] / 46, \mathrm{XX}[26]$ \\
314 & $45, \mathrm{X}[36] / 46, \mathrm{XY}, \mathrm{YP}+[24]$ \\
375 & $46, \mathrm{XY}, \mathrm{Yp}+$ \\
428 & $45, \mathrm{XY},-13,-19,+\operatorname{der}(19) \mathrm{t}(13 ; 19)(13 \mathrm{q} 12 ; 19 \mathrm{p} 13)$ \\
Severely oligozoospermic (No.) & \\
35 & $46, \mathrm{XY}, \mathrm{t}(14 ; \mathrm{Y})(\mathrm{p} 11 ; \mathrm{q} 11)$ \\
52 & $46, \mathrm{XY}, \mathrm{t}(4 ; 9)(\mathrm{q} 35 ; \mathrm{q} 12)$ \\
\hline
\end{tabular}

Table 3. Array-comparative genomic hybridization (CGH) analyses of three abnormal karyotype cases.

\begin{tabular}{|c|c|c|c|c|}
\hline No. & Karyotype & $\begin{array}{l}\text { Array-CGH result: } \\
\text { annotation hg18 }\end{array}$ & Size/gene & Note \\
\hline \multicolumn{5}{|c|}{ Azoospermic } \\
\hline 314 & $45, \mathrm{X}[36] / 46, \mathrm{X}, \mathrm{Yp}+[24]$ & $\begin{array}{l}\text { arr Xpterqter(6,329- } \\
154,895,334) \times 1\end{array}$ & None & $\begin{array}{l}\text { Only one X-chromosome; } \\
\text { Y-chromosome loss } \\
\text { (no Y material recognized } \\
\text { by array); poor coverage } \\
\text { for SRY gene, unclear } \\
\text { whether deleted }\end{array}$ \\
\hline 375 & $46, \mathrm{X}, \mathrm{Yp}+$ & $\begin{array}{l}\text { arr Ypterp11.31 } \\
(6,329-1,836,867) x 0, \\
\text { Yp11.31qter(1,839, } \\
629-57,758,621) \times 2\end{array}$ & $\begin{array}{l}\text { Size of the deletion Y pterp11.31: } \\
1.3 \mathrm{Mb} / \mathrm{SRY} \text { is gained }\end{array}$ & $46, \mathrm{X}, \mathrm{i}(\mathrm{Y})(\mathrm{p} 11.31)$ \\
\hline \multicolumn{5}{|c|}{ Severely oligozoospermic } \\
\hline 35 & $46, \mathrm{XY}, \mathrm{t}(14 ; \mathrm{Y})(\mathrm{p} 11 ; \mathrm{q} 11)$ & $\begin{array}{l}\text { arr Yq11.21q11.22(14, } \\
216,222-14,599,760) \times 2\end{array}$ & $\begin{array}{l}\text { Size of the gain Yq11.21q22.11: } \\
383.5 \mathrm{~kb} \text { including TMSB4Y gene }\end{array}$ & \\
\hline
\end{tabular}

Table 4. Microdeletion type analysis.

\begin{tabular}{lccccc}
\hline Phenotype & AZFa deletion \% $(\mathrm{N})$ & AZFb deletion \% $(\mathrm{N})$ & AZFc deletion \% $(\mathrm{N})$ & $\mathrm{AZFb}+\mathrm{c}$ deletion \% $(\mathrm{N})$ & Total deletion \% (N) \\
\hline $\begin{array}{l}\text { Azoospermic } \\
\begin{array}{l}\text { Severely } \\
\text { oligozoospermic }\end{array}\end{array}$ & $0.2(1 / 456)$ & $0.4(2 / 456)$ & $7.5(34 / 456)$ & $3.1(14 / 456)$ & $11.2(51 / 456)$ \\
\hline
\end{tabular}

Table 5. Analysis of abnormal karyotypes and sequence-tagged site (STS) deletion in azoospermic patients with
microdeletions.
\begin{tabular}{rlll}
\hline No. & Deletion No. & Deletion STS & Abnormal karyotype \\
\hline 72 & 6 & $\mathrm{sY} 127, \mathrm{sY} 134, \mathrm{sY} 152, \mathrm{sY} 157, \mathrm{sY} 254, \mathrm{sY} 255$ & $46, \mathrm{XX}[19] / 47, \mathrm{XXdel}(\mathrm{Y})(\mathrm{q} 11)[41]$ \\
98 & 9 & $\mathrm{sY} 127, \mathrm{sY} 134, \mathrm{sY} 152, \mathrm{sY} 157, \mathrm{sY} 254, \mathrm{sY} 255$ & $45, \mathrm{X},-\mathrm{Y},-15,+\mathrm{t}(\mathrm{Y} ; 15)(\mathrm{p} ? ; \mathrm{q} 11)$ \\
167 & 17 & $\mathrm{sY} 127, \mathrm{sY} 152, \mathrm{sY} 157, \mathrm{sY} 254, \mathrm{sY} 255$ & $46, \mathrm{XY}, \mathrm{del}(\mathrm{Yq})$ \\
241 & 26 & $\mathrm{sY} 127, \mathrm{sY} 152, \mathrm{sY} 157, \mathrm{sY} 254, \mathrm{sY} 255$ & $46, \mathrm{XY}[51] / 45, \mathrm{X}[9](\mathrm{Y}<\mathrm{G})$ \\
\hline
\end{tabular}




\section{DISCUSSION}

Although it has long been recognized that Y-chromosome microdeletions and chromosomal anomalies are closely related to male infertility, few studies have specifically examined the general characteristics of these Y-chromosome or the correlations between them in nonobstructive azoospermic and severely oligozoospermic infertile men in northeastern China.

The frequency and type of Y-chromosome microdeletions may be associated with ethnic and regional differences (Krausz et al., 2003). The frequency of Y-chromosome microdeletions varies widely among populations, ranging from 1.3 to 55.5\% (Osterlund et al., 2000; Foresta et al., 2001; Chiang et al., 2004). This large range of variation may be due to ethnic and regional differences, selection criteria for patient samples, or methodological differences. One study found no Y-chromosome microdeletions in non-obstructive azoospermic men in Croatia (Europe; Medica et al., 2005). Severely oligozoospermic men in West China, India, Taiwan (Asia), and Morocco (Africa) also display no Y-chromosome microdeletions (Lin et al., 2002; Imken et al., 2007; Mitra et al., 2008; Yang et al., 2008). However, our sample from northeastern China showed a higher frequency of microdeletions among severely oligozoospermic than among non-obstructive azoospermic men.

Five studies reported the highest frequency of microdeletions in the AZFc region (Foresta et al., 2000; Le Bourhis et al., 2000; Lin et al., 2000; Martinez et al., 2000; Tsujimura et al., 2004); the next highest frequency in 3 of these studies was found in the AZFb region (Foresta et al., 2000; Lin et al., 2000; Martinez et al., 2000) and in the AZFa region in 1 study (Le Bourhis et al., 2000). Additionally, non-obstructive azoospermic men from Japan (Asia), Brazil (South America), and Morocco (Africa) had no AZFb deletions, and non-obstructive azoospermic men from Brazil (South America) had no AZFc deletions [17-21,26-29] (Lin et al., 2002; Tsujimura et al., 2004; Medica et al., 2005; Ferlin et al., 2007; Imken et al., 2007; Mitra et al., 2008; Yang et al., 2008; Mirfakhraie et al., 2010; Mafra et al., 2011). Most microdeletions in our samples of patients from northeastern China occurred in the AZFc region (7.5\%), followed by the AZFb+c (3.1\%), AZFb $(0.4 \%)$, and AZFa $(0.2 \%)$ regions. Furthermore, we found AZFa and AZFb deletions in azoospermic men but not in severely oligozoospermic men.

The selection of an appropriate combination of STS loci is critical in the determination of Y-chromosome microdeletion frequency. The European Academy of Andrology and the European Molecular Genetics Quality Network guidelines indicate that the use of six STS loci (sY84, sY86, sY127, sY134, sY254, sY255) results in the detection of up to $95 \%$ of all reported Y-chromosome microdeletions in the AZF regions (Simoni et al., 2004). Additional STS loci can then be used to define the deletion breakpoints further. Certain microdeletion patterns may be related to the origin of the population (Peterlin et al., 2004), such as the deletions of sY240 and sY129 in the Japanese population (Kato et al., 2001) and sY269 and GY6 in the Italian population (Foresta et al., 2000). Although AZFd is known to be part of the AZFc region, a previous study in Japan (Tsujimura et al., 2004) used sY145, sY152, and sY153 to represent STS in the AZFd region. Our study also used sY152 and found a higher frequency $(91.5 \%)$ of sY152 microdeletions in infertile patients, followed by frequencies of sY254 and sY255. All of the severely oligozoospermic men had sY152 deletions. The frequency (21.6\%) of multiple segmental deletions in our sample was high among non-obstructive azoospermic patients with Y-chromosome microdeletions, all of whom had sY152, sY254, and sY255 de- 
letions in the $\mathrm{AZFb}+\mathrm{c}$ regions. Three of these patients also had sY127 and sY134 deletions; 4 also had sY127, sY134, and sY157 deletions; 3 also had sY127 and sY157 deletions; and 1 also had sY134 and sY157 deletions. Eight severely oligozoospermic patients also showed consecutive deletions (sY152, sY254, sY255) in the AZFc region; 7 of these patients also had sY157 deletions.

We found abnormal karyotypes in $10.2 \%$ of our study samples. We did not take Ychromosome polymorphism into account. This frequency agrees with a previously reported range of 2.2 to $14.3 \%$ for infertile men (Vicdan et al., 2004; Vutyavanich et al., 2007). The most common chromosomal anomaly in our samples was Klinefelter's syndrome (47,XXY; 77.4\%). Nine of the azoospermic patients had special chromosomal anomalies, of which 3 had chromosomal mosaicism and 6 had structural aberrations. Two severely oligozoospermic patients had special chromosomal anomalies, both translocations.

To the best of our knowledge, few studies have investigated the relationship between Y-chromosome microdeletions and chromosomal anomalies, two frequent genetic causes of spermatogenic failure. Four (7.8\%) of the 51 azoospermic patients with microdeletions had chromosomal anomalies, and all were multiple segmental deletions in the $\mathrm{AZFb}+\mathrm{c}$ regions. Two of these patients showed chromosomal mosaicism. One patient with mosaic Klinefelter's syndrome had a karyotype of $46, \mathrm{XX} / 47, \mathrm{XXdel}(\mathrm{Y})(\mathrm{q} 11)$ with a ratio of 19:41, and the other had a karyotype of $46, \mathrm{XY} / 45, \mathrm{X}(\mathrm{Y}<\mathrm{G})$ with a ratio of 51:9. The other 2 karyotypes were 45,X,-Y,-15,+t(Y;15)(p?;q11) and 46,XY,del(Yq). Our analyses have found novel correlations between Y-chromosome microdeletions and chromosomal anomalies, particularly Y-chromosome multiple segmental microdeletions and special chromosomal anomalies. The 2 cases of chromosomal mosaicism, 1 translocation, and 1 deletion have, to date, been found only in patients from northeastern China. Further studies should be conducted to confirm these correlations and mechanisms through the investigation of related cases.

A young man with oligozoospermia is likely to become azoospermic gradually. One of the patients in our study was oligozoospermic 1 year ago and has now developed azoospermia. This patient had microdeletions in the AZFc region (see Table 2, No. 46). This case indicates that men with AZFc deletions may produce mature spermatozoa, but that an age-dependent reduction in quantity may occur. This development may be addressed through cryopreservation of the spermatozoa of oligozoospermic young men. Although a high percentage of infertile men with Y-chromosome microdeletions are unable to produce children through natural reproductive mechanisms, assisted reproduction techniques may result in the transmission of the father's infertility problems to his sons. The detection of Y-chromosome microdeletions in infertile men is thus absolutely necessary.

In conclusion, the incidence of Y-chromosome microdeletions in the study population of infertile men from northeastern China was in accordance with that found in other populations, but the frequencies and locations of microdeletion sites differed. A peculiar finding in the northeastern China sample was the higher frequency of microdeletions among severely oligozoospermic men compared to non-obstructive azoospermic men. We discovered multiple segmental deletions in azoospermic patients with Y-chromosome microdeletions. We also found a correlation between Y-chromosome multiple segmental microdeletions and chromosomal anomalies, but further research is necessary to confirm these correlations and their mechanisms. These results emphasize the importance of genetic counseling and screening for Y-chromosome microdeletions and chromosomal anomalies for infertile men from northeastern China and for those in other regions to facilitate the development of appropriate treatment plans. 


\section{ACKNOWLEDGMENTS}

Research was generously supported by the Office of Science and Technology, Jilin Province, China (\#20080444-3).

\section{REFERENCES}

Balaresque P, Bowden GR, Parkin EJ, Omran GA, et al. (2008). Dynamic nature of the proximal AZFc region of the human Y chromosome: multiple independent deletion and duplication events revealed by microsatellite analysis. Hum. Mutat. 29: 1171-1180.

Balkan M, Tekes S and Gedik A (2008). Cytogenetic and Y chromosome microdeletion screening studies in infertile males with Oligozoospermia and Azoospermia in Southeast Turkey. J. Assist. Reprod. Genet. 25: 559-565.

Briton-Jones C and Haines CJ (2000). Microdeletions on the long arm of the Y chromosome and their association with male-factor infertility. Hong Kong Med. J. 6: 184-189.

Chiang HS, Yeh SD, Wu CC, Huang BC, et al. (2004). Clinical and pathological correlation of the microdeletion of Y chromosome for the 30 patients with azoospermia and severe oligoasthenospermia. Asian J. Androl. 6: 369-375.

Devroey P, Fauser BC and Diedrich K (2009). Approaches to improve the diagnosis and management of infertility. Hum. Reprod. Update 15: 391-408.

Ferlin A, Arredi B, Speltra E, Cazzadore C, et al. (2007). Molecular and clinical characterization of Y chromosome microdeletions in infertile men: a 10-year experience in Italy. J. Clin. Endocrinol. Metab. 92: 762-770.

Foresta C, Ferlin A and Moro E (2000). Deletion and expression analysis of AZFa genes on the human Y chromosome revealed a major role for DBY in male infertility. Hum. Mol. Genet. 9: 1161-1169.

Foresta C, Moro E and Ferlin A (2001). Y chromosome microdeletions and alterations of spermatogenesis. Endocr. Rev. 22: 226-239.

Gatta V, Stuppia L, Calabrese G, Morizio E, et al. (2002). A new case of Yq microdeletion transmitted from a normal father to two infertile sons. J. Med. Genet. 39: E27.

Imken L, El HB, Chafik A, Nahili H, et al. (2007). AZF microdeletions and partial deletions of AZFc region on the Y chromosome in Moroccan men. Asian J. Androl. 9: 674-678.

Kato H, Komori S, Nakata Y, Sakata K, et al. (2001). Screening for deletions in interval D16-22 of the Y chromosome in azoospermic and oligozoospermic Japanese men. J. Hum. Genet. 46: 110-114.

Kent-First M, Muallem A, Shultz J, Pryor J, et al. (1999). Defining regions of the Y-chromosome responsible for male infertility and identification of a fourth AZF region (AZFd) by Y-chromosome microdeletion detection. Mol. Reprod. Dev. 53: 27-41.

Kihaile PE, Yasui A and Shuto Y (2005). Prospective assessment of Y-chromosome microdeletions and reproductive outcomes among infertile couples of Japanese and African origin. J. Exp. Clin. Assist. Reprod. 2: 9.

Krausz C, Rajpert-De ME, Frydelund-Larsen L, Quintana-Murci L, et al. (2001). Double-blind Y chromosome microdeletion analysis in men with known sperm parameters and reproductive hormone profiles: microdeletions are specific for spermatogenic failure. J. Clin. Endocrinol. Metab. 86: 2638-2642.

Krausz C, Forti G and McElreavey K (2003). The Y chromosome and male fertility and infertility. Int. J. Androl. 26: 70-75.

Le Bourhis C, Siffroi JP, McElreavey K and Dadoune JP (2000). Y chromosome microdeletions and germinal mosaicism in infertile males. Mol. Hum. Reprod. 6: 688-693.

Lin YM, Chen CW, Sun HS, Hsu CC, et al. (2000). Y-chromosome microdeletion and its effect on reproductive decisions in taiwanese patients presenting with nonobstructive azoospermia. Urology 56: 1041-1046.

Lin YM, Lin YH, Teng YN, Hsu CC, et al. (2002). Gene-based screening for Y chromosome deletions in Taiwanese men presenting with spermatogenic failure. Fertil. Steril. 77: 897-903.

Mafra FA, Christofolini DM, Bianco B, Gava MM, et al. (2011). Chromosomal and molecular abnormalities in a group of Brazilian infertile men with severe oligozoospermia or non-obstructive azoospermia attending an infertility service. Int. Braz. J. Urol. 37: 244-250.

Martinez MC, Bernabe MJ, Gomez E, Ballesteros A, et al. (2000). Screening for AZF deletion in a large series of severely impaired spermatogenesis patients. J. Androl. 21: 651-655.

Medica I, Gligorievska N, Prenc M and Peterlin B (2005). Y microdeletions in the Istria county, Croatia. Asian J. Androl. 7: 213-216.

Mirfakhraie R, Mirzajani F, Kalantar SM, Montazeri M, et al. (2010). High prevalence of AZFb microdeletion in Iranian 
patients with idiopathic non-obstructive azoospermia. Ind. J. Med. Res. 132: 265-270.

Mitra A, Dada R, Kumar R, Gupta NP, et al. (2008). Screening for Y-chromosome microdeletions in infertile Indian males: utility of simplified multiplex PCR. Ind. J. Med. Res. 127: 124-132.

Organization WH (1999). Laboratory Manual for the Examination of Human Semen and Semen-Cervical Mucus Interaction. Cambridge University Press, Cambridge.

Osterlund C, Segersteen E, Arver S and Pousette A (2000). Low number of Y-chromosome deletions in infertile azoospermic men at a Swedish andrology centre. Int. J. Androl. 23: 225-229.

Peterlin B, Kunej T and Hristovski D (2004). Diagnostic test for Y chromosome microdeletion screening in male infertility. Genet. Test. 8: 45-49.

Shaffer LG, Slovak ML and Campbell LJ (2009). ISCN 2009: An International System for Human Cytogenetic Nomenclature, Karger.

Simoni M, Bakker E and Krausz C (2004). EAA/EMQN best practice guidelines for molecular diagnosis of y-chromosomal microdeletions. State of the art 2004. Int. J. Androl. 27: 240-249.

Tiepolo L and Zuffardi O (1976). Localization of factors controlling spermatogenesis in the nonfluorescent portion of the human Y chromosome long arm. Hum. Genet. 34: 119-124.

Tsujimura A, Matsumiya K, Takao T, Miyagawa Y, et al. (2004). Clinical analysis of patients with azoospermia factor deletions by microdissection testicular sperm extraction. Int. J. Androl. 27: 76-81.

Vicdan A, Vicdan K, Gunalp S, Kence A, et al. (2004). Genetic aspects of human male infertility: the frequency of chromosomal abnormalities and Y chromosome microdeletions in severe male factor infertility. Eur. J. Obstet. Gynecol. Reprod. Biol. 117: 49-54.

Vutyavanich T, Piromlertamorn W, Sirirungsi W and Sirisukkasem S (2007). Frequency of Y chromosome microdeletions and chromosomal abnormalities in infertile Thai men with oligozoospermia and azoospermia. Asian J. Androl. 9: 68-75.

Yang Y, Ma MY, Xiao CY, Li L, et al. (2008). Massive deletion in AZFb/b+c and azoospermia with Sertoli cell only and/ or maturation arrest. Int. J. Androl. 31: 573-578. 